\title{
EXCITED-STATE REACTION DYNAMICS OF BACTERIORHODOPSIN STUDIED BY FEMTOSECOND SPECTROSCOPY
}

\author{
J. DOBLER, W. ZINTH, W. KAISER \\ Physik Department E11, Technische Universität München, Munich, Federal Republic of Germany
}

and

D. OESTERHELT

Max-Planck-Institut für Biologie, Martinsried/Munich, Federal Republic of Germany

Received 4 January 1988

\begin{abstract}
The photodynamics of bacteriorhodopsin were studied by transient absorption and gain measurements after excitation with femtosecond pulses at $620 \mathrm{~nm}$. With probing pulses at longer wavelengths $(\lambda>770 \mathrm{~nm})$ the previously reported formation of the $J$ intermediate (with a time constant of $500 \pm 100 \mathrm{fs}$ ) was confirmed. With probing pulses around $700 \mathrm{~nm}$, a faster process with a relaxation time of $200 \pm 70$ fs was observed. The data analysis strongly suggests that this kinetic constant describes the reactive motion of the polyatomic molecule on its excited-state potential energy surface, i.e. one observes directly the incipient isomerization of the retinal molecule. The minimum of the $S_{1}$ potential energy surface reached in $200 \mathrm{fs}$ lies approximately $13300 \mathrm{~cm}^{-1}$ above the ground state of bacteriorhodopsin and from this minimum the intermediate $\mathbf{J}$ is formed with a time constant of $500 \mathrm{fs}$.
\end{abstract}

\section{Introduction}

Photosynthetic activity in halobacteria is based on the membrane protein bacteriorhodopsin (BR), which acts as a light-driven proton pump. BR contains only one chromophore, a retinal molecule, which is linked via a protonated Schiff's base to lysine 216 in the primary structure of the protein. In the functionally active light-adapted form of BR the retinal molecule adopts the all-trans configuration. The photochemical reaction of BR has been studied intensively during the past decade [1], but only recently, the primary reaction leading to the first ground-state photoproduct could be resolved [2-5]. Light quanta promote the BR molecule to the first excited electronic state $\left(S_{1}\right)$. From the bottom of the $S_{1}$ potential surface the transition to the photoproduct $J$ takes place within 430 fs and a subsequent reaction leads to the intermediate photoproduct $\mathrm{K}$ with a time constant of 3 ps [4-6] ${ }^{\# 1}$. The primary re-

\#1 In ref. [4] we published a time constant of 5 ps for the $J$ to $K$ transition. Our more recent femtosecond experiments indicate a somewhat shorter time constant of $3 \pm 1$ ps. action is an all-trans to 13-cis [7], presumably 13cis, 14s-cis photoisomerization [8]. In the current interpretation the reactive motion during the initial steps proceeds on the $S_{1}$ potential surface as well as on the ground-state $\left(S_{0}\right)$ surface. Until now, no timeresolved experimental observation of the excited-state reactive motion has been reported.

Wc apply improved femtosecond time-resolved methods to the study of the excited-state dynamics of bacteriorhodopsin. Transient absorption and gain experiments are described which reveal the motion of the retinal molecule on its excited-state potential energy surface.

\section{Material and methods}

Bacteriorhodopsin (as purple membranes) was prepared from Halobacterium halobium according to the published procedure [9]. Samples had optical densities of $20-30$ at $\lambda=570 \mathrm{~nm}$ and were placed in a rotating cell of $300 \mu \mathrm{m}$ optical path. The BR sample was kept in the light-adapted form by appropri- 
ate background illumination. Time-resolved excite and probe experiments were performed using $80 \mathrm{fs}$ pulses from a colliding pulse mode-locked (CPM) laser/amplifier system operated at a repetition rate of $7 \mathrm{kHz}[10,11] .10 \%$ of the output of the laser/ amplifier system served as the exciting pulse. The excitation wavelength was $\lambda=620 \mathrm{~nm}$ and the excitation energy $200 \mathrm{~nJ}$. Approximately $10 \%$ of the BR molecules within the illuminated area were excited by each pulse. The main part of the energy of the laser output generated a broad continuum in a $1 \mathrm{~mm}$ thick ethylene glycol jet [12]. A small fraction of the femtosecond continuum of $10 \mathrm{~nm}$ width selected via interference filters gave the probing light pulse. The time resolution of the experiment was determined by the cross-correlation function between exciting and probing pulses. The cross-correlation widths $\tau_{\text {. }}$ (fwhm) slightly depended on the selected wavelength of the probing pulses. A typical value was $\tau_{c}=120 \mathrm{fs}$ at $\lambda=850 \mathrm{~nm}$.

Transmission changes of the sample induced by the excitation pulses were recorded as a function of time. In order to guarantee a continuously fresh sample in the experiment the cuvette $(8 \mathrm{~cm}$ diameter) rotated at $12.5 \mathrm{~Hz}$. In this way each pulse illuminated a new portion of the sample and after one revolution the intermediate photoproducts were reconverted to the starting condition, i.e. the lightadapted BR.

\section{Results}

Fig. 1 shows the absorption and fluorescence of light-adapted BR. The excitation and probing wavelengths are indicated by arrows. The absorption band is broad and the excitation wavelength of $\lambda=620 \mathrm{~nm}$ lies on its red wing. At wavelengths $\lambda>650 \mathrm{~nm}$ residual absorption is smaller than required to explain the absorbance decrease seen in fig. 2 by simply bleaching the ground-state absorption. The fluorescence band is also broad. It peaks around $730 \mathrm{~nm}$ and decays slowly towards the near infrared; at 850 $\mathrm{nm}$ the fluorescence intensity exhibits $50 \%$ of its peak value.

Results of the time-resolved experiments are presented in fig. 2 for four probing wavelengths. The experimental results are shown as circles; the solid curves are calculated using a simplified level scheme, where excitation light populates a molecular state with reduced absorption. This level decays with the time constant $\tau=200$ fs to a further species of increased absorption (figs. $2 a$ and $2 b$ ). The curves in figs. $2 c$ and $2 d$ are calculated using a decay time $\tau$ of

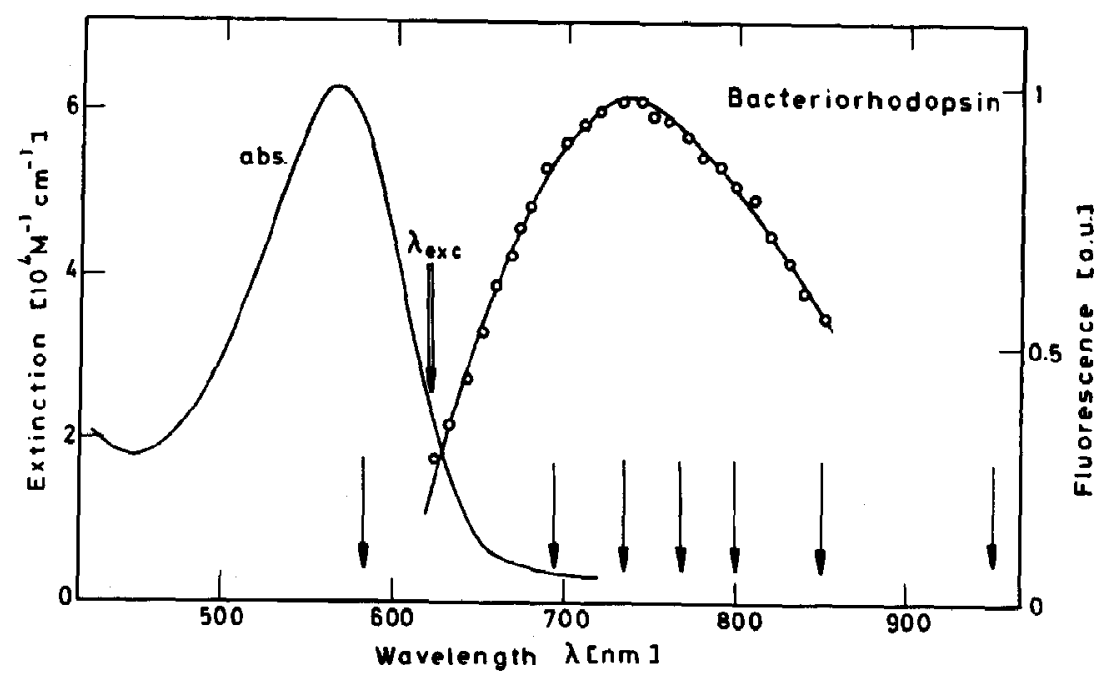

Fig. 1. Absorption and fluorescence emission spectra of light-adapted bacteriorhodopsin. The excitation and probing wavelengths of the femtosecond experiments are indicated by the arrows. 


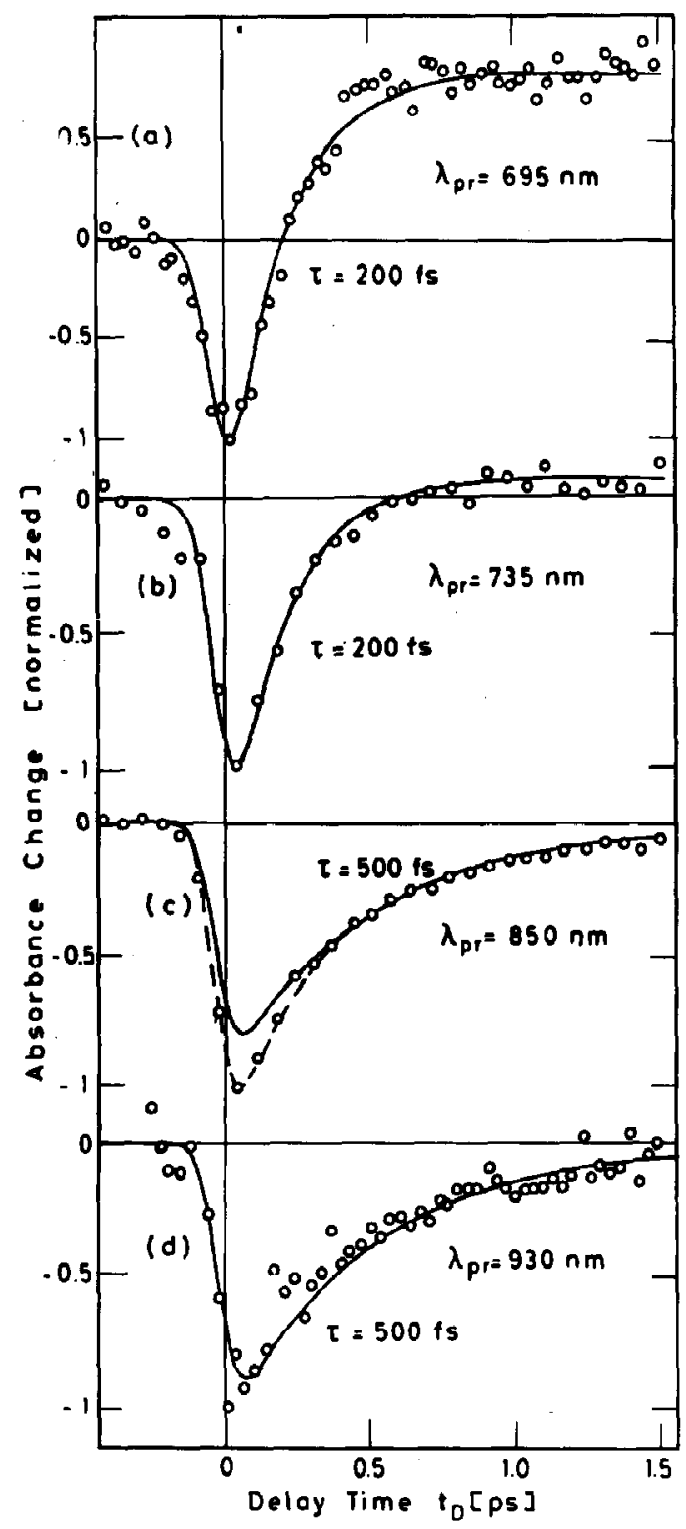

Fig. 2. Time-resolved absorption induced by exciting femtosecond pulses at $\lambda=620 \mathrm{~nm}$ detected at probing wavelengths $\lambda=695$ $\mathrm{nm}$ (a), $735 \mathrm{~nm}$ (b), $850 \mathrm{~nm}$ (c) and $930 \mathrm{~nm}$ (d). The experimental points are normalized to the peak absorption changes. The solid curves are calculated using the model given in the text with decay times of $200 \mathrm{fs}(\mathrm{a}, \mathrm{b})$ or $500 \mathrm{fs}(\mathrm{c}, \mathrm{d})$. The broken curve takes into account a double-exponential decay with both time constants.

500 fs and a negligibly increased absorption at later times. Each curve is normalized to the maximal changes, which correspond to transmission changes of the order of several percent. At the shortest wavelength, $\lambda=695 \mathrm{~nm}$ (fig. $2 \mathrm{a}$ ), a rapid reduction of the absorption to negative values around time zero turns with a time constant of 200 fs to a level of enhanced absorption. Because BR has no appreciable absorption at $\lambda=695 \mathrm{~nm}$ (see fig. 1 ), the observed negative absorption at short delay times must be due to light gain by the excited BR molecule. At a probing wavelength of $\lambda=735 \mathrm{~nm}$ (as well as at $695 \mathrm{~nm}$ ) (fig. 2b) the early gain disappcars with a time constant of 200 fs and a small increased absorption due to the product states appears concomitantly. At $850 \mathrm{~nm}$ (fig. 2c) a gain decay kinetics with $\tau=500 \mathrm{fs}$ is measured ( solid curve). A small contribution of the $200 \mathrm{fs}$ process explains the differences between the experimental data points and the calculated (solid) curve. A further red-shift of the probing light pulse to $930 \mathrm{~nm}$ makes the 200 fs process undetectable and the gain curve can be fitted by a single 500 fs time constant (fig. 2d). Measurements were taken at $\lambda=770$ and $800 \mathrm{~nm}$ but are not shown in fig. 2 . At $770 \mathrm{~nm}$ a double-exponential decay is composed of contributions from the 200 and 500 fs kinetics with equal amplitudes. The data at $800 \mathrm{~nm}$ can be fitted by the same time constants with an amplitude contribution of the 200 fs process of approximately $25 \%$.

When the same experiments were performed in the absorption region of BR, e.g. at $585 \mathrm{~nm}$, an absorption region of BR, e.g. at $585 \mathrm{~nm}$, an absorbance decrease recovered in $500 \pm 100$ fs. No distinct contribution from a faster component was detected. This result is in agreement with our previous observation at $\lambda=620 \mathrm{~nm}$ of a $430 \pm 50 \mathrm{fs}$ decay time [2].

The observed absorption changes are related to population changes of the ground state $S_{0}$ and the excited state $S_{1}$ of $B R$. Considering the momentary population densities $N_{0}$ of $\mathrm{S}_{0}$ and $N_{1}$ of $\mathrm{S}_{1}$, three processes are of interest here: (i) absorption from $S_{0}$ proportional to $N_{0}$; (ii) excited-state absorption from $S_{1}$ proportional to $N_{1}$; (iii) stimulated emission (amplification or gain) from $\mathrm{S}_{\mathrm{t}}$ proportional to $N_{1}-N_{0}$.

\section{Discussion}

\subsection{General consideration}

Before presenting a model for the primary reac- 
tion in BR we summarize our experimental observations. We find: (i) negative absorption (amplification or gain) with a relaxation time of 200 fs for probing wavelengths between 695 and $850 \mathrm{~nm}$ (energy between 14400 and $11800 \mathrm{~cm}^{-1}$ ); (ii) negative absorption with a lifetime of $500 \mathrm{fs}$ for probing wavelengths $\lambda>735 \mathrm{~nm}$ (the onset of the $500 \mathrm{fs}$ component is between 735 and $770 \mathrm{~nm}$, i.e. the high-frequency limit is between 13600 and $13000 \mathrm{~cm}^{-1}$, (iii) two time constants of 200 and $500 \mathrm{fs}$ in the overlapping wavelength range of (i) and (ii).

The following interpretation takes advantage of current knowlege on fast processes on excited electronic surfaces. The potential energy surfaces of the retinal chromophore of bacteriorhodopsin for the ground state $S_{0}$ and for the first excited state $S_{1}$ are shown as a function of reaction coordinate in fig. 3 . The latter is related to the isomerization of the retinal molecule.

During excitation with the pump pulse the retinal chromophore is excited to Franck-Condon states on the $S_{1}$ potential surface. These states are rapidly depopulated with the time constant $\tau_{1}$ by reactive motion along the $S_{1}$ potential surface. As a result of this

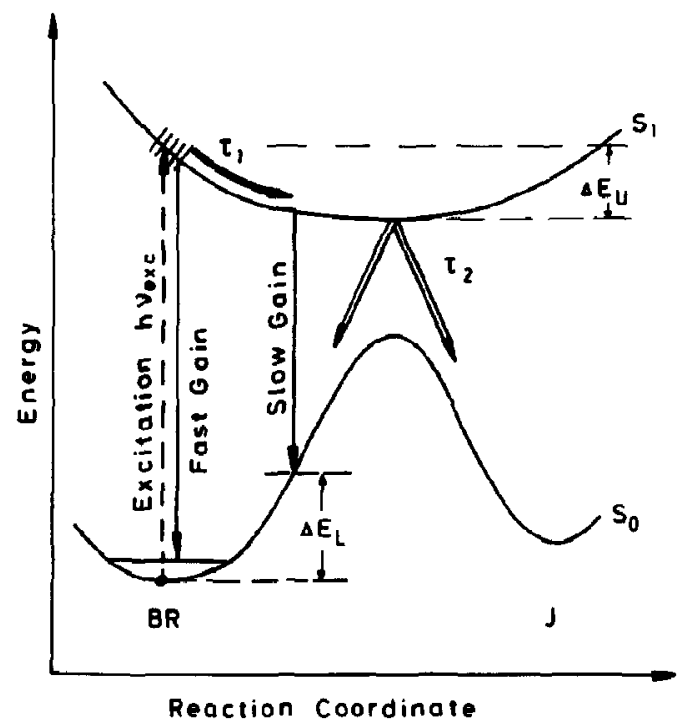

Fig. 3. Model of the ground-state $\left(S_{0}\right)$ and excited $\left(S_{1}\right)$ energy surfaces as a function of the reaction coordinate. The first motion leads the system with the time constant of $200 \mathrm{fs}$ to the bottom of the $S_{1}$ potential surface. The reaction proceeds to the ground-state photoproduct $J$ or the initial state of BR with the time constant of $500 \mathrm{fs}$. fast motion the gain from the Franck-Condon states (fig. 3 ) is very short-lived, $\tau_{1}=200$ fs. The relaxation from the minimum of the $S_{1}$ potential surface has a longer time constant, $\tau_{2}=500 \mathrm{fs}$, and is seen as a slow gain at smaller energies $(\lambda>770 \mathrm{~nm})$.

\subsection{The excitation process}

The excitation follows the Franck-Condon principle, i.e. the optical transition proceeds vertically without changing the nuclear coordinates of the molecule. The broad absorption spectrum (see fig. 1) and the steep slope of the $S_{1}$ potential surface (see below) suggests an inhomogeneously broadened transition (a wide $S_{0}$ potential minimum). Light at a certain wavelength is absorption by a number of ground-state configurations and a hole is burned in the corresponding population. This hole evolves in time with the thermal motion of the retinal molecules. The hole vanishes within a period of a low-lying vibrational level within 50-100 fs. On the other hand, the molecules on the $S_{1}$ potential surface are - immediately after excitation - in the Franck-Condon states, depicted schematically by the shaded area in fig. 3. At that point various normal modes are displaced from their equilibrium position on the $S_{1}$ potential surface. One can understand this state as a coherent superposition of different normal modes (a wave packet) [13]. The energy of this Franck-Condon state is higher than the bottom (equilibrium position) of the $S_{1}$ surface by $\Delta E_{u}$. Experimentally, at wavelengths close to the excitation wavelength $\left(\lambda_{\mathrm{pr}} \approx \lambda_{\text {exc }}\right)$ one observes both the depletion (hole) in the ground-state population $N_{0}$ and the change in the excited-state population $N_{1}$. Using shorter probing wavelengths, $\lambda_{\mathrm{pr}} \ll \lambda_{\text {exc }}$, the filling of the hole in the ground-state population $N_{0}$ and possible excited-state absorption $\left(N_{1}\right)$ from the Franck-Condon state are seen. 'At longer wavelengths, $\lambda_{\mathrm{pr}} \gg \lambda_{\mathrm{exc}}$, gain and excited-state absorption from the Franck-Condon state are observed (both proportional to $N_{1}$ ).

\subsection{Motions on the $S_{I}$ potential surface}

The evolution of the system after excitation may be described as follows. The initial wave packet consists of different low- to medium-frequency vibra- 
tional modes. The excited modes move the molecule along the reaction coordinate. No energy exchange of the retinylidene moiety, e.g. by collision with solutes, is possible within the protein matrix and thus the process proceeds undisturbed. The wave packet spreads out due to the rapid dephasing processes in the excited electronic state [14] and the energy stored in the Franck-Condon states of the molecules distributes over many degrees of freedom of the large retinal molecule. At the minimum of the potential energy surface most of the energy $\Delta E_{u}$ is quasi-thermalized. The system stays within an energy $k T^{*}$ at the bottom of the $S_{1}$ state. A transient temperature $T^{*}=400 \mathrm{~K}$ is estimated from the relaxed energy $\Delta E_{\mathrm{u}}$ [15].

A simple classical treatment allows us to estimate the reaction time from the Franck-Condon state to the relaxed state. We assume that an energy of $\Delta E_{\mathrm{u}} \approx 2500 \mathrm{~cm}^{-1}$ (see below) is available for the reactive motion. The initial reaction is believed to be a 13-cis double-bond rotation or a 13-cis, 14s-cis isomerization $[7,8,16,17]$. The initial rotation in both cases requires (for rotation angles up to $60^{\circ}$ ) a mass transfer which is equivalent to the movement of a group of atoms (consisting of one carbon, one nitrogen and several hydrogens) by less than $1.5 \AA$. The time $t$ necessary to move the atoms over a distance $x$ is estimated to be $t=\left(2 m x^{2} / \Delta E_{\mathrm{u}}\right)^{1 / 2} \#_{2}$. In the present case one calculates $t=200 \mathrm{fs}$. The exact agreement with the experimental short relaxation time must be considered fortuitous on account of the uncertainty of the different factors entering the estimate of $t$.

\subsection{The relaxed $S_{l}$ state}

At the minimum of the $S_{1}$ surface the system has access to a wide range of the reaction coordinate via thermal motions. Three major processes start from this relaxed state: Light emission or gain in a broad frequency range and internal conversion leading to the ground-state surfaces of either the initial state or the product state $\mathrm{J}[18]$.

We believe that the longer time constant of $500 \mathrm{fs}$ is related to the decay of this relaxed state. This interpretation is supported by the fact that the slow

\footnotetext{
${ }^{\# 2}$ A linear dependence of the potential energy $E(x)$ was assumed.
}

gain is at longer wavelengths than the fast gain (fig. 3 ). In addition, we note that the 500 fs process is the only slow gain process observed. Information on the $S_{1}$ potential surface is obtained from the following energy considerations:

$h \nu_{\mathrm{exc}}=E_{\mathrm{g}}+\Delta E_{\mathrm{L}}+\left(\Delta E_{\mathrm{u}}-k T^{*}\right)$.

Here $E_{\mathrm{g}}$ is the high-frequency limit of the slow gain, $E_{\mathrm{g}}=13000 \mathrm{~cm}^{-1}(770 \mathrm{~nm})$ and $\Delta E_{\mathrm{L}}$ marks the energy of the terminal state on the $S_{0}$ potential surface of the gain at $13000 \mathrm{~cm}^{-1}$. An upper limit of the energy $\Delta E_{\mathrm{u}}$ (dissipated during the $\mathrm{S}_{1}$ motion) mauy be calculated from the equation given above with the excitation energy $h v_{\mathrm{exc}}=16100 \mathrm{~cm}^{-1}$ and a lower limit for $\Delta E_{\mathrm{L}}$ of several $k T \approx 500 \mathrm{~cm}^{-1}$. With these numbers we estimate an upper limit of $\Delta E_{\mathrm{u}} \leqslant 2800$ $\mathrm{cm}^{-1}$.

A final remark should be added to the time-resolved experiments in the absorption region of $\mathrm{BR}$. In these experiments [2] BR was excited and probed at the same wavelength $\lambda=620 \mathrm{~nm}$ and no fast component was seen. The reduced time resolution $\left(t_{\mathrm{p}}=150 \mathrm{fs}\right)$ and the coherent artifact around time zero presumably prevented the detection of the faster component.

Interesting femtosecond experiments with improved time resolution have been perfomed by Mathies and Shank in the absorption region of BR around $620 \mathrm{~nm}$ [19]. Their time-resolved spectra are caused by transient population changes in the excited electronic state as well as in the ground state.

\section{Conclusion}

We followed the fast photochemical reaction of bacteriorhodopsin on the excited-state potential surface and observed the motion out of the initially populated Franck-Condon region proceeding in 200 fs. Subsequently, the system reaches a relaxed state at the minimum of the $S_{1}$ potential surface which decays in $500 \mathrm{fs}$. The experimental results indicate that only a small amount of energy $\Delta E_{\mathrm{u}}<2800 \mathrm{~cm}^{-1}$ is available for the excited-state reactive process. This finding places the bottom of the $S_{1}$ potential state at least $13300 \mathrm{~cm}^{-1}$ above the ground state of the lightadapted bacteriorhodopsin. 


\section{Acknowledgement}

The authors acknowledge valuable discussions with Drs. P. Tavan, R.A. Mathies, and C.V. Shank, who kindly informed us of their results prior to publication. The authors also thank M.A. Franz for supplying the fluorescence spectrum of $B R$.

\section{References}

[1] J.K. Lanyi, in: Bioenergetics, ed. L. Ernster (Elsevier, Amsterdam, 1984) p. 314;

W. Stoeckenius and R.A. Bogomolni, Ann. Rev. Biochem. 52 (1982) 587.

[2] M.C. Nuss, W. Zinth, W. Kaiser, E. Kölling and D. Oesterhelt, Chem. Phys. Letters 117 (1985) 1.

[3] A.V. Sharkov, A.V. Pakulev, S.V. Chekalin and Y.A. Matveetz, Biochim. Biophys. Acta 808 (1985) 94.

[4] H.J. Polland, M.A. Franz, W. Zinth, W. Kaiser, E. Kölling and D. Oesterhelt, Biophys. J. 49 (1986) 651.

[5] H.J. Polland, W. Zinth and W. Kaiser, in: Ultrashort phenomena, Vol. 4, eds. D.H. Auston and K.B. Eisenthal (Springer, Berlin, 1984) p. 456.

[6] J.W. Petrich, J. Breton, J.L. Martin and A. Antonetti, Chem. Phys. Letters 137 (1987) 369.

[7] S.O. Smith, I. Hornung, R. van der Steen, N.S. Braiman, J. Lugtenburg, A. Pardoen and R.A. Mathies, Proc. Natl. Acad. Sci. US 83 (1986) 967.
[8] K. Gerwert and F. Siebert, EMBO J. 5 (1986) 805; K. Schulten and P. Tavan, Nature 272 (1978) 85.

[9] D. Oesterhelt and W. Stoeckenius, Methods Enzymol. 31A (1974) 667.

[10] R.L. Fork, B.I. Greene and C.V. Shank, Appl. Phys. Letters 38 (1981) 671 .

[11] W.H. Knox, M.C. Downer, R.L. Fork and C.V. Shank, Opt. Letters 9 (1984) 552.

[12] R.L. Fork, C.V. Shank, R.T. Yen, C. Hirlimann and W.J. Tomlinson, in: Picosecond phenomena, Vol. 3, eds. K.B. Eisenthal, R.M. Hochstrasser, W. Kaiser and A. Laubereau (Springer, Berlin, 1982) p. 10.

[13] A.B. Myers and R.A. Mathies, in: Biological applications of Raman spectroscopy, Vol. 2, ed. T.A. Spiro (Wiley, New York, 1987) p. 1.

[14] A.M. Weiner, S. de Silvestri and E.P. Ippen, J. Opt. Soc. Am. B 2 (1985) 654.

[15] A. Seilmeier, P.O.J. Scherer and W. Kaiser, Chem. Phys. Letters 105 (1984) 140.

[16] G.S. Harbison, S.O. Smith, J.A. Pardoen, C. Winkel, J. Lugtenburg, J. Herzfeld, R.A. Mathies and R.G. Griffin, Proc. Natl. Acad. Sci. US 81 (1984) 1706.

[17] M. Engelhard, K. Gerwert, B. Hess, W. Kreutz and F. Siebert, Biochemistry 24 (1985) 400.

[18] A. Warshel and M. Karplus, Chem. Phys. Letters 32 (1975) 11.

[19] R.A. Mathies and C.V. Shank, presentation at the Symposium on Spectroscopy on Retinal Proteins, Freiburg (September 1987). 